\title{
Análise do consumo alimentar de Universitários de uma Instituição Privada de Ensino Superior em São Luís-MA
}

\section{Analysis of the food consumption of university students at a private higher education institution in São Luís-MA}

Ellen Mila Cutrim Maciel ${ }^{1}$, Naylanne Lima de Sousa ${ }^{2}$, Juliane Wend Vieira Silva ${ }^{3}$, Janaina Maiana Abreu Barbosa ${ }^{4}$, Virginia Nunes Lima ${ }^{5}$

\section{RESUMO}

O consumo alimentar de universitários é influenciado por diversos fatores, destacam-se a percepção sobre alimentação saudável, questão econômica e disponibilidade de tempo para preparar e realizar refeições, podendo ser visto como um futuro problema de saúde pública. Com isso, a pesquisa teve por objetivo, avaliar o consumo alimentar e a percepção sobre alimentação saudável de universitários de uma universidade privada em São Luís- MA. Trata-se de estudo analítico e transversal com 192 universitários, utilizando Questionário de Frequência Alimentar, questionário socioeconômico e questionário sobre percepção de alimentação saudável. Da amostra total, $78,65 \%$ pertenciam ao gênero feminino, com idade entre 18 a 66 anos e média de 22 anos. O consumo alimentar diário dessa população caracterizou-se pela alta prevalência de alimentos considerados não saudáveis como bebidas com adição de açúcar $(66,1 \%)$ e baixo consumo de alimentos considerados saudáveis como frutas $(32,8 \%)$, legumes e hortaliças $(29,2 \%)$, carnes e ovos $(29,7 \%)$. O conhecimento nutricional desta população, classificou-se como moderado $(61,98 \%)$. Com isso, ficou perceptível a necessidade de atividades informativas e educativas relacionadas a promoção da alimentação saudável dentro do ambiente universitário, como ferramenta para promoção de hábitos saudáveis a longo prazo.

Palavras-chave: Alimentação Saudável. Saúde Coletiva. Nutrição.

\section{ABSTRACT}

The food consumption of university students is influenced by several factors, including the perception of healthy eating, economic issues and the availability of time to prepare and eat meals, which can be seen as a future public health problem. Thus, the research aimed to assess food consumption and the perception of healthy eating among university students at a private university in São Luís-MA. This is an analytical and crosssectional study with 192 university students, using the Food Frequency Questionnaire, socioeconomic questionnaire and questionnaire on the perception of healthy eating. Of the total sample, $78.65 \%$ belonged to the female gender, aged between 18 and 66 years and an average of 22 years. The daily food consumption of this population was characterized by the high prevalence of foods considered unhealthy as drinks with added sugar $(66.1 \%)$ and low consumption of foods considered healthy, such as fruits (32.8\%), vegetables $(29.2 \%)$, meat and eggs $(29.7 \%)$. The nutritional knowledge of this population was classified as moderate $(61.98 \%)$. As a result, the need for informative and educational activities related to the promotion of healthy eating within the university environment was perceived as a tool for promoting healthy habits in the long term.
Nutricionista CEUMA .

ORCID: 7951-2153

E-mail: mylacutrim16@gmail.com

Nutricionista CEUMA.

ORCID: https://orcid.org/00000002-0511-2330

3 Nutricionista - Universidade CEUMA

ORCID: https://orcid.org/00000002-6413-8020

${ }^{4}$ Nutricionista. Mestre em Saúde Coletiva pela Universidade Federal do Maranhão.

ORCID: 5263-6586

orcid.org/0000-0001-

${ }^{5}$ Nutricionista. Mestre em Gestão de Programas e Serviços de Saúde pela Universidade Ceuma.

Keywords: Healthy eating. Collective Health. Nutrition. 


\section{INTRODUÇAOO}

Ao longo das últimas décadas, a sociedade vem sofrendo uma impactante transição nutricional, marcada pela redução dos índices de desnutrição e aumento dos níveis de sobrepeso e obesidade, relacionados diretamente às alterações na qualidade da dieta e maior prevalência de Doenças Crônicas Não Transmissíveis (DCNT) (BARROS et al., 2018; CHRISTOFOLETTI et al., 2020).

A prevalência de morbidades está associada principalmente ao consumo alimentar habitual, como dietas ricas em gorduras saturadas que causam elevação dos valores séricos de colesterol e lipoproteínas de baixa densidade (LDL), consumo excessivo de alimentos industrializados, responsáveis pelo aumento na pressão arterial sistólica e ingestão elevada de ácidos graxos trans, considerados fatores de riscos para doenças cardiovasculares (DCV) (TEIXEIRA et al., 2018; OLIVEIRA et al., 2018; IANISKI et al., 2018; SANTOS; CABRAL, 2019).

Uma alimentação saudável e de qualidade é um dos principais aliados na prevenção e/ou tratamento de doenças crônicas, e corresponde ao consumo regular de alimentos naturais que se adequam às necessidades diárias da ingestão de nutrientes para garantia da qualidade de vida, correto desenvolvimento e crescimento fisiológico (SOUZA; SOUZA, 2017).

O consumo alimentar saudável é um direito humano básico e uma boa alimentação deve obedecer aos princípios da quantidade e qualidade, sendo eles a variedade de nutrientes, o equilíbrio energético e a diminuição do consumo de alimentos industrializados (JAIME et al., 2018; MENÊSES et al., 2017; MARCONATO; SILVA; FRASSON, 2016; LANZILLOTTI et al., 2019)

O conhecimento e percepção sobre alimentação saudável são fatores importantes na promoção e garantia da qualidade de vida, e principais determinantes na escolha por alimentos saudáveis. A percepção começa a ser formada desde a transição entre a infância e adolescência, e constitui-se na vida adulta (BUSATO, 2015).

$O$ ingresso na universidade tem se tornado um dos grandes responsáveis por alterações no hábito alimentar de acadêmicos, caracterizados principalmente pela omissão de refeições ou substituição por alimentos industrializados e de preparo rápido, como salgadinhos e refrigerantes, que são ricos em sódio, açúcares e gorduras saturadas, além da baixa prevalência na ingestão de alimentos in natura, como frutas e hortaliças, ricos em fibras, vitaminas e minerais (SOUZA; SOUZA, 2017). 
Além disso, situações como deixar a casa dos pais e passar a viver sozinhos, apresentar dificuldades em manipular e preparar suas refeições, ter novas relações sociais, estresse e preocupação com as diversas atividades a serem realizadas dentro do âmbito acadêmico, também prejudicam na escolha de alimentos mais saudáveis, e estes novos hábitos podem ser seguidos posteriormente a graduação gerando problemas a saúde dessa população (MIRANDA; CASTRO; SANTANA, 2016; SOUZA; SOUZA, 201).

Com isso, pensando na conduta alimentar dos universitários como um futuro problema de saúde pública, o presente estudo buscou analisar o consumo alimentar desta população e identificar sua percepção sobre uma alimentação saudável.

\section{MATERIAIS E METODOS}

Trata-se de um estudo analítico, transversal, realizado em uma universidade privada, localizada na cidade de São Luís - MA, no período compreendido entre abril a junho de 2018. Inicialmente, a pesquisa foi submetida e aprovado pelo Comitê de Ética em Pesquisa da Universidade Ceuma, sob o número de parecer 2.599.257.

A amostra foi composta por acadêmicos de ambos os sexos, com idade maior que 18 anos, dos cursos de graduação em Nutrição, Farmácia, Enfermagem, Fisioterapia, Fonoaudiologia, Odontologia e Educação física (bacharel e licenciatura), que aceitaram participar do estudo.

Foram coletados os dados socioeconômicos através da aplicação de um questionário adaptado do modelo utilizado pelo Instituto Federal de Educação Ciência e Tecnologia do Piauí (IFPI, 2014), contido nele perguntas fechadas sobre idade, gênero, estado civil, cor da pele auto referida, quantidade total de pessoas residentes no domicilio e renda familiar.

Procedeu-se também a investigação do consumo alimentar se utilizando do Questionário de Frequência Alimentar (QFA), retirado do estudo de Sichieri e Everhart (2002), que avalia através de perguntas referentes a frequência de consumo em número de vezes por dia, semana e mês e os seguintes grupos de alimentos: leites e derivados, carnes e ovos, óleos, petiscos e enlatados, cereais e leguminosas, frutas e hortaliças, sobremesas e doces, bebidas e produtos diet e light.

$\mathrm{Na}$ identificação da Percepção sobre Alimentação Saudável, foi utilizado a Escala de Conhecimento Nutricional, traduzida e validada por Scagliusi et al. (2006), com perguntas fechadas, referentes ao conhecimento geral sobre os alimentos, como: "Você já ouviu falar em fibras alimentares?", "Quantas porções de frutas e hortaliças você acha que uma pessoa deve comer por dia, para ter uma boa saúde?", "Você sabe o que são carboidratos?", "Onde 
você encontra os alimentos ricos em carboidratos?", "Você já ouviu falar em gorduras?", "Quais alimentos contém mais gordura?", que avaliou a percepção sobre alimentação saudável do indivíduo.

Para a classificação do conhecimento sobre alimentação saudável os resultados foram categorizados da seguinte maneira: 1 a 2 acertos das perguntas do questionário classificou-se como conhecimento regular; de 3 a 4 acertos como conhecimento moderado e 5 acertos como alto conhecimento nutricional.

Os dados numéricos foram colhidos e tabulados no programa Excel 2013, e avaliados pelo Software de estatística STATA 14.0 (STATA CORP COLLEGE STATION, TEXAS, $E \cup A$ ), foi realizado a análise descritiva, onde as variáveis qualitativas foram apresentadas por meio de frequências e porcentagens, já as variáveis quantitativas foram apresentadas por médias.

\section{RESULTADOS}

Participaram da pesquisa 192 acadêmicos dos cursos de graduação da área da saúde, onde 19,79\% eram do curso de nutrição, $18,23 \%$ de fisioterapia e $17,19 \%$ de odontologia (Tabela 1).

Em relação ao gênero houve predominância do sexo feminino (78,65\%). A idade dos universitários variou entre 18 a 66 anos e apresentou a média de 22 anos. Os universitários que mais se dispuseram a participar da pesquisa foram os que estavam cursando entre os três (3) primeiros períodos da graduação $(41,14 \%)$. Predominou a cor parda da pele auto referida $(57,81 \%)$ e branca $(26,56 \%)$.

A prevalência da renda familiar dos universitários foi entre 1 a 3 salários mínimos $(53,13 \%)$, a maioria ainda reside com os pais $(48,96 \%)$ e são solteiros $(93,75 \%)$. A maioria $(90,1 \%)$ também afirmaram que a vida acadêmica interferia direta ou indiretamente na alimentação habitual.

Tabela 1. Características socioeconômicas dos acadêmicos de uma universidade privada de São Luís-MA.

\begin{tabular}{ll}
\hline Características & $\mathbf{N}(\%)$ \\
\hline Cursos & $18(9,38)$ \\
Educação Física & $18(9,38)$ \\
Enfermagem & $28(14,58)$ \\
Farmácia & \\
\hline
\end{tabular}


A.; LIMA, V. N

Análise do consumo alimentar de Universitários de uma Instituição Privada de Ensino Superior em São Luís-MA

\section{Nutrição \\ Sexo \\ Período

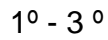 \\ $4 \stackrel{\circ}{-6} \div$

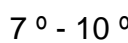

Fisioterapia

Fonoaudiologia

Odontologia

Masculino (1)

Feminino (2)

Raça

Branca

Negra

Parda

Amarela

Indígena

Renda familiar

$<1$ Salário Min.

1 - 3 Salários Min.

4 - 6 Salários Min.

7 - 10 Salários Min.

>10 Salários Min.

\section{Com quem reside}

Pais

Parentes

Amigos

Sozinho

\section{Estado civil}

Solteiro

Casado

Interferência da vida acadêmica na alimentação

Sim

Não

\section{$35(18,23)$}

$22(11,46)$

$38(19,79)$

$33(17,19)$

$41(21,35)$

$151(78,65)$

$79(41,15)$

$67(34,89)$

$46(23,96)$

$51(26,56)$

$27(14,06)$

$111(57,81)$

$2(1,04)$

$1(0,52)$

$8(4,17)$

$102(53,13)$

$53(27,60)$

$22(11,46)$

$7(3,65)$

$94(48,96)$

$52(27,08)$

$11(5,73)$

$55(18,23)$

$180(93,75)$

$12(6,25)$

$173(90,10)$

$19(9,90)$

Fonte: Construção da autora.

A prevalência da ingestão de alimentos saudáveis pelos acadêmicos estudados está representada na Tabela 2 . Entre os alimentos saudáveis mais consumidos diariamente 
estão arroz, pães e massas (72,9\%), seguido por leites e derivados (69,3\%). A prevalência de consumo semanal, tem destaque para as carnes e ovos $(45,83)$, legumes e hortaliças (45,31\%), já os alimentos que são consumidos em uma frequência menor, de 1 a 3 vezes por mês são os tubérculos $(27,1 \%)$. Neste estudo foi possível observar que o consumo de alimentos in natura prevalece semanalmente, enquanto que o consumo diário desses alimentos é considerado baixo, comparado à recomendação do Guia alimentar para a população brasileira (BRASIL, 2014).

Tabela 2. Características do consumo de alimentos considerados saudáveis de universitários de uma Universidade privada de São Luís-MA.

\begin{tabular}{|c|c|c|c|c|c|c|c|}
\hline \multirow{3}{*}{$\begin{array}{l}\text { Grupo de } \\
\text { alimento }\end{array}$} & \multirow{3}{*}{ Porção } & \multicolumn{6}{|c|}{ Frequência } \\
\hline & & Nunca & $\begin{array}{l}1 \text { a } 2 \text { vezes } \\
\text { por semana }\end{array}$ & $\begin{array}{l}5 \text { a } 6 \text { vezes } \\
\text { por semana }\end{array}$ & $\begin{array}{l}2 \text { a } 4 \text { vezes } \\
\text { por semana }\end{array}$ & $\begin{array}{l}1 \text { vez por } \\
\text { semana }\end{array}$ & $\begin{array}{c}1 \text { a } 3 \text { vezes } \\
\text { por mês }\end{array}$ \\
\hline & & $\mathrm{N}(\%)$ & $\mathrm{N}(\%)$ & $\mathrm{N}(\%)$ & $\mathrm{N}(\%)$ & $\mathrm{N}(\%)$ & $\mathrm{N}(\%)$ \\
\hline $\begin{array}{l}\text { Leites e } \\
\text { derivados }\end{array}$ & 1 & $2(1,04)$ & $133(69,3)$ & $16(8,33)$ & $33(17,19)$ & $6(3,13)$ & $2(1,04)$ \\
\hline Carnes e ovos & 2 & $0(0)$ & $57(29,7)$ & $45(23,44)$ & $88(45,83)$ & $2(1,04)$ & $0(0)$ \\
\hline $\begin{array}{l}\text { Óleos e } \\
\text { gorduras }\end{array}$ & 1 & $2(1,04)$ & $114(59,5)$ & $21(10,94)$ & $41(21,35)$ & $8(4,17)$ & $6(3,13)$ \\
\hline $\begin{array}{l}\text { Arroz, pães e } \\
\text { massas }\end{array}$ & 1 & $1(0,5)$ & $140(72,9)$ & $12(6,25)$ & $33(17,19)$ & $3(1,56)$ & $3(1,6)$ \\
\hline $\begin{array}{c}\text { Grãos } \\
\text { integrais }\end{array}$ & 1 & $137(71,3)$ & $28(14,6)$ & $4(2.08)$ & $12(6,25)$ & $3(1,56)$ & $8(4,2)$ \\
\hline Leguminosas & 1 & $8(4,2)$ & $44(22,9)$ & $21(10,94)$ & $84(43,75)$ & $21(10,94)$ & $14(7,3)$ \\
\hline $\begin{array}{l}\text { Legumes e } \\
\text { hortaliças }\end{array}$ & 1 & $8(4,2)$ & $56(29,2)$ & $18(9,38)$ & $87(45,31)$ & $18(9,38)$ & $5(2,6)$ \\
\hline Tubérculos & 1 & $5(2,6)$ & $16(8,3)$ & $7(3,65)$ & $43(22,40)$ & $27(14,06)$ & $52(27,1)$ \\
\hline Frutas & 1 & $6(3,1)$ & $25(32,8)$ & $31(16,15)$ & $69(35,94)$ & $11(5,73$ & $12(6,2)$ \\
\hline $\begin{array}{l}\text { Bebidas sem } \\
\text { açúcar }\end{array}$ & 1 & $138(71,9)$ & $24(12,5)$ & $4(2,08)$ & $14(7,29)$ & $7(3,65)$ & $5(2,6)$ \\
\hline
\end{tabular}

Fonte: Construção da autora.

Entre os alimentos considerados não saudáveis, a prevalência no consumo diário de café/bebidas com açúcar é de $66,1 \%$, seguido pelo consumo de alimentos do grupo dos óleos como manteiga/margarina/maionese $(59,4 \%)$. No que se refere ao consumo desses alimentos com a frequência semanal, houve destaque para o consumo dos doces $(31,25 \%)$ e mensalmente os lanches (25,5\%), como pode ser observado na Tabela 3. 
Tabela 3. Consumo de alimentos considerados não saudáveis dos universitários de uma Universidade privada em São Luís-MA.

\begin{tabular}{|c|c|c|c|c|c|c|c|}
\hline \multirow{3}{*}{$\begin{array}{l}\text { Grupo de } \\
\text { alimento }\end{array}$} & \multirow{3}{*}{ Porção } & \multicolumn{6}{|c|}{ Frequência } \\
\hline & & Nunca & $\begin{array}{l}1 \text { a } 2 \text { vezes } \\
\text { por semana }\end{array}$ & $\begin{array}{c}5 \text { a } 6 \text { vezes } \\
\text { por } \\
\text { semana }\end{array}$ & $\begin{array}{l}2 \text { a } 4 \text { vezes } \\
\text { por semana }\end{array}$ & $\begin{array}{l}1 \text { vez por } \\
\text { semana }\end{array}$ & $\begin{array}{c}1 \text { a } 3 \\
\text { vezes por } \\
\text { mês }\end{array}$ \\
\hline & & $\mathrm{N}(\%)$ & $\mathrm{N}(\%)$ & $\mathrm{N}(\%)$ & $\mathrm{N}(\%)$ & $\mathrm{N}(\%)$ & $\mathrm{N}(\%)$ \\
\hline Embutidos & 1 & $44(23)$ & $13(6,7)$ & $7(3,65)$ & $51(26,56)$ & $33(17,19)$ & $44(22,9)$ \\
\hline Lanches & 1 & $20(10,4)$ & $14(7,4)$ & $3(1,56)$ & $55(28,65)$ & $51(26,56)$ & $49(25,5)$ \\
\hline Doces & 1 & $8(4,2)$ & $41(21,3)$ & $16(8,33)$ & $60(31,25)$ & $37(19,27)$ & $30(15,6)$ \\
\hline $\begin{array}{l}\text { Bebidas com } \\
\text { adição de } \\
\text { açúcar }\end{array}$ & 1 & $10(5,2)$ & $127(66,1)$ & $10(5,21)$ & $31(16,15)$ & $6(3,13)$ & $8(4,2)$ \\
\hline $\begin{array}{c}\text { Produtos diet } \\
\text { e light }\end{array}$ & 1 & $140(72,9)$ & $22(11,5)$ & $4(2,08)$ & $11(5,73)$ & $5(2,60)$ & $10(5,2)$ \\
\hline
\end{tabular}

Fonte: Construção da autora.

Verificou-se que a percepção sobre alimentação saudável dos acadêmicos teve uma predominância do nível classificado como moderado (61,98\%), no entanto, apenas 12,50\% atingiram o alto nível de conhecimento nutricional (Figura 1).

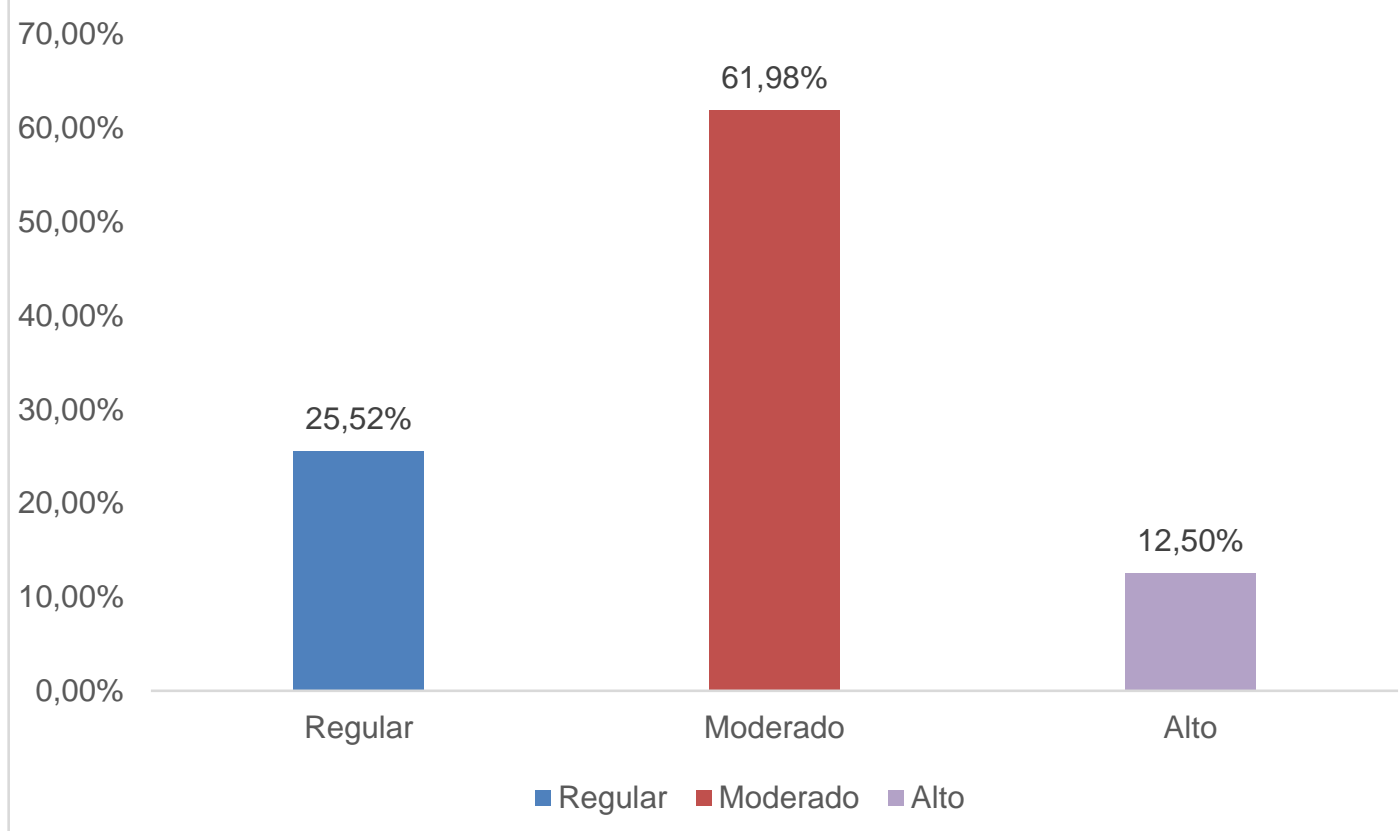

Figura 1. Classificação da percepção sobre alimentação saudável dos universitários em uma universidade em São Luís- MA. 


\section{DISCUSSĀO}

A análise socioeconômica demonstrou similaridade aos resultados encontrados no estudo de Oliveira et al. (2017) que avaliaram o perfil sociodemográfico, nutricional e alimentar de 173 estudantes do curso de nutrição de uma universidade pública em LagartoSE, identificando predominância do gênero feminino (84\%) e raça auto declarada, parda (58\%). Kanematsu et al. (2016), analisou o conceito de alimentação saudável entre 42 universitários de uma Universidade Estadual do Centro-Oeste, onde houve maior participação do gênero feminino $(95,2 \%)$, destacando-se o curso de nutrição $(23,8 \%)$ e maioria dos entrevistados estavam nos períodos iniciais da graduação $(57,1 \%)$.

Já Souza e Souza (2017) quando avaliaram o hábito alimentar de 225 universitários de uma Instituição privada de ensino superior do interior da Bahia, também observaram a prevalência do sexo feminino $(82,2 \%)$, porém em relação a renda familiar, houve divergência do presente estudo, com maior citação no percentual para 2-4 salários mínimos $(60,9 \%)$.

Em relação ao consumo de alimentos considerados saudáveis, Almeida et al. (2017) quando analisaram a qualidade da alimentação como fator de risco para câncer de intestino de 100 universitários do curso de Gastronomia de uma instituição de ensino privada em Montes Claros, encontraram valores dissimilares ao presente estudo, onde no consumo diário houve destaque para alimentos como: arroz polido (54\%), feijão (68\%) carne bovina (29\%) e frango (33\%).

Neste estudo, foi possível notar que o consumo diário de leites e seus derivados foram predominantemente altos, resultado dissemelhante foram encontrados por Crepaldi et al. (2016) ao avaliarem a prevalência de fatores de risco para doenças crônicas não transmissíveis entre estudantes, ao qual $68,6 \%$ dos entrevistados apresentavam baixo consumo de leites. Os autores ainda citam a importância do consumo frequente de produtos lácteos e derivados, visto a sua associação a menor prevalência ao risco de doenças crônicas.

Ahmed e Ahmed (2015), avaliaram o consumo de alimentos de 367 acadêmicos da Universidade de Dammam, na Arábia Saudita, e concluiu-se que a maioria dos participantes $(72,20 \%)$ da pesquisa tinham conhecimento sobre as recomendações da Organização Mundial da Saúde (OMS) em relação ao consumo diário de frutas e hortaliças, porém não consumiam a quantidade desejada desses grupos de alimentos. 
Como visto na Tabela 2, o consumo diário de frutas, legumes e hortaliças foram menores em comparação ao consumo de alimentos não saudáveis, como as bebidas com adição de açúcar. No estudo de Amaral et al. (2017), onde foi realizada a avaliação da alimentação de recém ingressos nos cursos de nutrição e ciências biológicas de uma universidade pública de Juiz de Fora, ao qual o consumo de verduras e legumes foram relatados por $43,75 \%$, salada crua por $59,82 \%$ e frutas por $65,18 \%$ dos universitários como consumo diário, resultados estes, superiores ao presente estudo.

No estudo de Oliveira et al. (2015), os autores avaliaram os hábitos alimentares de 254 acadêmicos do curso de nutrição no Maranhão, foi constatado que acadêmicos do último ano do referido curso consumiam mais alimentos saudáveis como frutas, hortaliças e laticínios, em comparação aos graduandos do primeiro ano do curso, esse resultado foi distinto ao do presente estudo, onde se observou que o consumo alimentar de acadêmicos do curso de nutrição não se diferiu dos demais cursos, apesar de se ter identificado que a percepção sobre alimentação saudável é mais relevante comparado aos demais cursos, fato que pode ser justificado pela abordagem de disciplinas especificas do curso.

Segundo Honorato et al. (2019), caracteriza-se por alimentação saudável a associação de refeições saudáveis, balanceadas, variadas e equilibradas em nutrientes e que sejam compostas em sua maioria por alimentos in natura, e ofereçam benefícios na promoção da saúde. O consumo diário de frutas e verduras, segundo o Ministério da Saúde deve ser no mínimo 3 porções ao dia para um bom aporte de nutrientes, no presente estudo este consumo diário ficou bem abaixo das recomendações da OMS (BRASIL, 2014).

Com o ingresso na universidade, o consumo alimentar de jovens sofre grandes modificações, seja pela mudança da casa dos pais, excesso de atividades acadêmicas stress, falta de tempo, modismos dietéticos ou falta de manejo no preparo de refeições, influenciam para um maior consumo de alimentos industrializados, de fácil preparo ou prontas, como fast foods e até mesmo omissão de algumas refeições (SOUZA; SOUZA, 2017; HONORATO et al., 2019).

Para Oliveira et al. (2019) as frequentes desculpas pela "falta de tempo" contribuem para um maior consumo de alimentos considerados não saudáveis, dentre eles alimentos processados e ultraprocessados, com baixa qualidade nutricional e ricos em açúcar, gorduras e sódio.

Quando analisados os alimentos considerados não saudáveis, Aquino; Pereira e Reis (2015), encontraram resultados semelhantes ao presente estudo quanto ao consumo semanal de doces entre os estudantes estava elevado (96\%), e a alta prevalência no 
consumo de lanches como hambúrguer, salgados, batata chips e pizzas $(93,5 \%)$, outro aspecto a ser considerado é a frequência do consumo de bebidas com adição de açúcar que teve relevância semanalmente $(62,66 \%)$ divergente ao encontrado na atual pesquisa.

Voser et al., (2018) ao analisarem o perfil alimentar e conhecimento nutricional de jogadores universitários de futsal, encontram resultados semelhante ao deste estudo em relação ao consumo do grupo de doces, onde houve uma frequência no consumo de $33,05 \%$.

Alkaed et al. (2018), validaram um questionário sobre conhecimento nutricional (KANLQ), com a participação de 253 universitários do Kuwait, e constataram que estudantes da área da saúde e principalmente do curso de graduação em nutrição tiveram um resultado significativo com ótimo conhecimento $(79,84 \%)$ que indicou uma boa validade para a construção do questionário. Resultado que corrobora com o conhecimento nutricional verificado no presente estudo onde o nível de conhecimento está de acordo com o curso.

\section{CONSIDERACOOEES FINAIS}

Os resultados encontrados demonstraram que a frequência de consumo semanal dos alimentos saudáveis, como carnes e ovos, legumes, hortaliças e frutas, é mais prevalente correlacionado ao consumo diário, sendo considerado baixo de acordo com recomendações da OMS. Já no grupo dos alimentos não saudáveis, o consumo diário das bebidas com adição de açúcar se sobressai, em seguida está o consumo de doces, ou seja, a alimentação desta população é rica em açúcares simples.

Sobre a percepção de alimentação saudável dos universitários, conclui-se que o nível de conhecimento é moderado.

Com isso, foi possível observar a necessidade de atividades educativas voltadas para a promoção da alimentação saudável dentro do ambiente universitário, buscando estimular e educador sobre hábitos saudáveis como garantia de saúde.

\section{REFERENCIAS}

AHMED, A. A.; AHMED, B. Fruit and vegetable consumption and its determinants among Saudi university students. Journal of Taibah University Medical Sciences, v. 10, n. 2, p. 201-207, 2015. https://www.sciencedirect.com/science/article/pii/S1658361214001255 
ALKAED, D. et al. Validity and Reliability of a Nutrition Knowledge Questionnaire in an Adult Student Population. Journal of Nutrition Education and Behavior, v. 50, n. 7, p. 718-723, 2018. https://pubmed.ncbi.nlm.nih.gov/29705510/

ALMEIDA, L. et al. Alimentação como fator de risco para câncer de intestino em universitários. Revista Brasileira em Promoção da Saúde, Fortaleza, v. 30, n. 1, p. 72 78, 2017. https://periodicos.unifor.br/RBPS/article/view/5882/pdf

AMARAL, V. S. et al. avaliação da alimentação de recém ingressos nos cursos de nutrição e ciências biológicas em uma universidade pública. Revista Multiverso, v. 2, n. 1, p. 4957, 2017. http://periodicos.jf.ifsudestemg.edu.br/multiverso/article/view/128

AQUINO, J. K.; PEREIRA, P.; REIS, V. M C. P. Hábito e consumo alimentar de estudantes do curso de nutrição das faculdades de Montes Claros - Minas Gerais. Revista Multitexto, $\begin{array}{llllll}\text { v. } 3, & \text { n. } & 01, & \text { p. } & 82-88, & \end{array}$ http://www.ead.unimontes.br/multitexto/index.php/rmcead/article/view/111

BARROS, G. G. M. et al. Transição nutricional e sua relação com a prevalência de hipertensão arterial em índios brasileiros. Revista Diálogos Acadêmicos, Fortaleza, v. 7, n. 2, P. 20-23, 2018. http://revista.fametro.com.br/index.php/RDA/article/view/187/188

BRASIL. Ministério da Saúde. Secretaria de Atenção Básica. Guia alimentar para a população brasileira. 2 ed. 2014 . Disponível em: https://bvsms.saude.gov.br/bvs/publicacoes/guia alimentar populacao brasileira 2ed.pdf. Acesso em: set. 2019.

BUSATO, M. A. et al. Ambiente e alimentação saudável: percepções e práticas de estudantes universitários. Seminal: Ciências Biológicas e da Saúde, Londrina, v. 36, n. 2, p. 75-84, 2015. http://www.uel.br/revistas/uel/index.php/seminabio/article/view/21447

CHRISTOFOLETTI, M. et al. Simultaneidade de doenças crônicas não transmissíveis em 2013 nas capitais brasileiras: prevalência e perfil sociodemográfico. Epidemiol. Serv. Saude, Brasília, v. 29, n. 1, p. $1-12,2020$. https://www.scielo.br/scielo.php?script=sci arttext\&pid=S2237-96222020000100308

CREPALDI, B. V. C. et al. Elevada prevalência de fatores de risco para doenças crônicas entre universitários. Ciência e Saúde, v. 9, n. 3, p. 135-143, 2016. https://revistaseletronicas.pucrs.br/ojs/index.php/faenfi/article/view/22938

HONORATO, A. C. S. et al. Avaliação da qualidade de vida e consumo de frutas e verduras de universitários. Revista Brasileira de Obesidade, Nutrição e Emagrecimento, São Paulo, v.13, n. 81, p.776-781, 2019. http://www.rbone.com.br/index.php/rbone/article/view/1061

IANISKI, V. B. et al. Estilo de vida associado a níveis séricos de HDL colesterol em longevos: Estudo AMPAL. Revista Kairós-Gerontologia, São Paulo, v. 21, n. 3, p. 111127, 2018. https://ken.pucsp.br/kairos/article/view/41415

JAIME, P. C. et al. Um olhar sobre a agenda de alimentação e nutrição nos trinta anos do Sistema Único de Saúde. Ciência e Saúde Coletiva, v. 23, n. 6, p. 1829-1836, 2018. 
https://www.scielo.br/scielo.php?pid=S1413$81232018000601829 \&$ script $=$ sci abstract\&tlng $=$ pt

KANEMATSU, L. R. A. et al. Conceito de Alimentação Saudável: Análise das Definições Utilizadas por Universitários da Área da Saúde. UNICIÊNCIAS, v. 20, n. 1, p.34-38, 2016. https://revista.pgsskroton.com/index.php/uniciencias/article/view/4343

LANZILLOTTI, H. S. et al. Estimativa do padrão alimentar de estudantes de Nutrição de uma universidade estadual no Rio de Janeiro, Brasil. DEMETRA, Rio de Janeiro, v. 14, p. 1-21, 2019. https://www.e-publicacoes.ueri.br/index.php/demetra/article/view/35717

MENÊSES, L. E. N. et al. Consumo alimentar e estado nutricional de crianças em uma escola privada de Palmas, Tocantins. Revista Desafios, v. 4, n. 3, p. 43-51, 2017. https://sistemas.uft.edu.br/periodicos/index.php/desafios/article/view/3223/9964

MIRANDA, A. S.; CASTRO, J. S.; SANTANA, R. F. Perfil Nutricional e Alimentar de Universitárias do Sudoeste da Bahia. UNICIÊNCIAS, v. 20, n. 1, p. 39-43, 2016. https://doi.org/10.17921/1415-5141.2016v20n1p39-43

OLIVEIRA, A. C. et al. Hábitos alimentares de acadêmicas do primeiro e do último ano de cursos de graduação em Nutrição no estado do Maranhão. Nutrire, v. 40, n. 3, p. 328-336, 2015. http://sban.cloudpainel.com.br/files/revistas publicacoes/480.pdf

OLIVEIRA, J. S. et al. Avaliação do perfil sociodemográfico, nutricional e alimentar de estudantes de nutrição de uma universidade pública em Lagarto-SE. RASBRAN, São Paulo, v. 8, n. 2, p. 37-42, 2017. https://www.rasbran.com.br/rasbran/article/view/465

OLIVEIRA, M. R. M. et al. Insegurança alimentar e nutricional e fatores de risco para doenças crônicas não transmissíveis em catadores de resíduos sólidos. Demetra, v. 13, n. 3, $\quad$ p. $\quad 635-647, \quad 2018.2$ https://www.epublicacoes.ueri.br/index.php/demetra/article/view/34088

SANTOS, H. V. D.; CABRAL, P. C. Consumo alimentar e níveis de colesterol sérico em mulheres nutricionistas. DEMETRA, Rio de Janeiro, v. 14, p. 1-14, 2019. https://www.epublicacoes.uerj.br/index.php/demetra/article/view/38710\#: : :text=A\%20maior\%20ingest\% C3\%A30\%20proteica\%20da,os\%20grupos\%20avaliados\%20neste\%20estudo.

SOUZA, M. T. A.; SOUZA, F. M. Avaliação dos hábitos alimentares dos Universitários de uma Instituição Privada de Ensino Superior no interior da Bahia. Id on line Revista

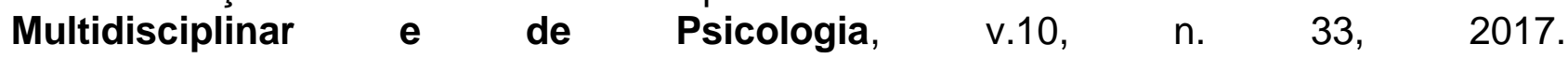
https://idonline.emnuvens.com.br/id/article/view/618

TEIXEIRA, V. B. D. et al. Transição nutricional no estado de minas gerais em 1974 e 2009: um estudo com enfoque na população idosa. Revista Brasileira de Educação e Cultura, n. $17, \quad$ p. 2018. https://periodicos.cesg.edu.br/index.php/educacaoecultura/article/view/344

VOSER, R. C. et al. Conhecimento nutricional, perfil alimentar e estado nutricional de jogadores universitários de futsal. Revista Brasileira de Nutrição Esportiva, São Paulo, v. 12, n. 71. p. 394-403, 2018. http://www.rbne.com.br/index.php/rbne/article/view/1059 